\title{
„Für Gerechten Handel mit der Dritten Welt”. Hintergründe und Forderungen einer Aktion schweizerischer Hilfswerke
}

Pour un commerce équitable avec le Tiers Monde:

Enjeux et objectifs d'une action lancée par des œuvres d'entraide suisses

\section{Richard Gerster}

\section{OpenEdition}

\section{Journals}

\section{Electronic version}

URL: http://journals.openedition.org/sjep/1073

DOI: $10.4000 /$ sjep.1073

ISSN: 1663-9677

Publisher

Institut de hautes études internationales et du développement

\section{Printed version}

Date of publication: 1 janvier 1986

Number of pages: 177-181

ISSN: 1660-5926

\section{Electronic reference}

Richard Gerster, « „Für Gerechten Handel mit der Dritten Welt”.

Hintergründe und Forderungen einer Aktion schweizerischer Hilfswerke », Schweizerisches Jahrbuch

für Entwicklungspolitik [Online], 6 | 1986, Online erschienen am: 09 März 2013, abgerufen am 08

September 2020. URL : http://journals.openedition.org/sjep/1073; DOI : https://doi.org/10.4000/sjep. 1073 


\title{
«FUER GERECHTEN HANDEL MIT DER DRITTEN WELT॥. HINTERGRUENDE UND FORDERUNGEN EINER AKTION SCHWEIZERISCHER HILFSWERKE
}

\author{
Richard Gerster
}

Die Aktion «Für gerechten Handel mit der Dritten Welt» wurde am 16. Januar 1986 gemeinsam von Brot für Brüder, Caritas, Fastenopfer, HEKS, Helvetas, Arbeiterhilfswerk und Swissaid gestartet. Die Entstehung der Aktion ist sowohl spezifisch schweizerischen Umständen als auch den allgemeinen weltwirtschaftlichen Perspektiven zuzuschreiben.

\section{WELTWIRTSCHAFTLICHE GROSSWETTERLAGE}

Die meisten Entwicklungsländer befinden sich mitten in einer schweren Wirtschaftskrise und die Zukunftsperspektiven sind alles andere als rosig.

- Die Rohstoffpreise sind seit 1980 auf breiter Front gesunken. Der "Economist» schätzte, dass die OECD-Staaten 1985 für den RohstoffEinkauf rund 65 Milliarden Dollar weniger aufwenden mussten als im Vorjahr. Die überschuldeten Entwicklungsländer stehen unter dem Zwang, um jeden Preis Devisen zu verdienen - und reagieren auf fallende Weltmarktpreise oft mit Produktionssteigerungen, was wiederum den Druck auf die Preise erhöht. Im Aussenwirtschaftsbericht 1985 hat der Bundesrat unmissverständlich festgehalten: "Die Lage im Rohstoffsektor, die sich auf die internationale Entwicklungszusammenarbeit auswirkt, gibt zu grosser Besorgnis Anlass.»

- Sinn und Zweck von Rohstoffabkommen wurden noch nie so massiv in Frage gestellt wie heute. Einerseits hängt dies mit der allgemeinen Renaissance marktwirtschaftlicher Vorstellungen zusammen, welche interventionistischen Rohstoffregelungen - wenn überhaupt - nur einen bescheidenen Stellenwert zugesteht. Anderseits hat der Zusammenbruch des Zinnmarktes die Grenzen konventioneller Rohstoffabkommen klar signalisiert. Der gemeinsame Rohstoffonds ist mangels genügender Ratifikationen gar nicht in Kraft getreten - Zeichen der bereits seit Jahren schleichenden Krise in der Rohstoffdiplomatie. Die ruinöse Konkurrenz mancher Rohstoffproduzenten verunmöglicht heute von vornherein jegliche Marktregelung auf der Basis von Produktionsbeschränkungen. 
- Die Industrieländer bauen ihre Handelsbarrieren an der Grenze aus und schützen so ihre Landwirtschaft und Industrie vor unliebsamer Konkurrenz. Dieser «Freihandel von Fall zu Fall» beraubt gerade hoch verschuldete Entwicklungsländer der Möglichkeit, Einnahmen aus dem Export zu erwirtschaften und aus der Schuldenfalle herauszufinden, ohne lebensnotwendige Importe kürzen zu müsen. Im Industriebereich weist die Schweiz wohl eine weisse Weste auf, doch ist nach Ansicht von Hans J. Mast (Schweizerische Kreditanstalt) "der schweizerische Agrar-Protektionismus vielleicht nach Japan der extremste der Welt».

Dieses weltwirtschaftliche Wetterleuchten lenkt einmal mehr die Aufmerksamkeit auf die Grenzen der Entwicklungszusammenarbeit im engeren Sinn hin. Denn die Früchte der Projektarbeit wachsen in einem entwicklungsfreundlichen Wirtschaftsklima am besten.

\section{SCHWEIZERISCHE AUSGANGSLAGE}

Das Vorfeld der Aktion «Für gerechten Handel mit der Dritten Welt» in der Schweiz war durch drei Umstände charakterisiert:

- Grenzen der Dritte-Welt-Laden-Bewegung werden spürbar. Rund zehn Jahre sind es her, seit die Hilfswerke OS3, die Import-und Informationsstelle für Waren aus Entwicklungsgebieten, gegründet haben. OS3 beliefert über 500 Dritte Welt-Läden und Verkaufsstellen in der ganzen Schweiz, welche sich um gerecht bezahlte und umweltschonend hergestellte Erzeugnisse aus Entwicklungsländern bemühen. Das ist ein Erfolg, der sich sehen lassen darf - und trotzdem ist nicht zu verkennen, dass diese Bewegung heute an Grenzen stösst. Grenzen für viele Lieferanten aus Entwicklungsländern, welche uns mehr Produkte verkaufen möchten.

- Gespräche mit Wirtschaftskreisen haben bisher keinen Durchbruch entwicklungspolitischer Anliegen in kommerziellen Kanälen gebracht. Im Zusammenhang mit der Lieferung von Ananas aus den Philippinen durch Del Monte ist der Versuch entstanden, in die Lieferverträge für den Schweizer Markt eine Sozialklausel einzubauen. Eine Ueberprüfung der Situation steht bevor. Mit den Grossverteilern Migros/ Coop wurden Gespräche geführt, sozial- und umweltgerechte Produkte aus Entwicklungsländern mit einem entwicklungspolitischen Gütezeichen auszuzeichnen, das dem Konsumenten eine rasche Orientierung im Laden erlaubt. Die Gespräche wurden schliesslich sistiert, weil die Verteiler - einmal abgesehen von praktischen Durchführungsproblemen letzlich nicht an einen für sie interessanten Markt glaubten - im Gegensatz zu den Hilfswerken.

- Ein Konsumenteninformationsgesetz näherte sich der parlamentarischen Phase. Dieses Gesetz kann die Grundlagen legen für eine ausreichende Produkteinformation betreffend Herkunft und Herstellbedingun- 
gen. Eine verpflichtenden Produkteinformation fällt jedoch nicht vom Himmel, sondern bedarf einer starken Lobby.

\section{ZIELSETZUNGEN DER AKTION}

Mit der Charakterisierung des wirtschaftlichen und politischen Umfeldes werden auch die Ziele der Aktion «Für gerechten Handel mit der Dritten Welt» offenbar:

- Ueber die Projekthilfe hinausweisen und die Bedeutung des weltwirtschaftlichen Rahmens für die Dritte Welt bewusst machen ;

- In Information über sozial gerechte und umweltkonforme Produkte aus Entwicklungsländern investieren im Hinblick darauf, dass daraus ein kommerziell interessanter Teilmarkt entsteht;

- Auf die Notwendigkeit einer unvermindert aktiven Rohstoffdiplomatie mit entwicklungspolitischen Vorzeichen aufmerksam machen und diesbezügliche schweizerische Bemühungen stützen ;

- Gegenüber dem Parlament die Erwartungen breiter Bevölkerungsschichten an das neue Konsumenteninformationsgesetz bezüglich Produktedeklaration dokumentieren.

Nur mit einem breit angelegten Massnahmenbündel konnten diese Zielsetzungen verfolgt werden. Kernstück stellt der gleichnamige Appel dar, für den während neun Monaten Unterschriften gesammelt worden sind.

\section{FORDERUNGEN DES APPELS «FUER GERECHTEN HANDEL MIT DER DRITTEN WELT»}

Aus den Zielsetzungen wiederum entstehen Forderungen an die Konsumenten, die Wirtschaft, die Behörden und die Parlamentarier. Der Unterzeichner des Appels geht in seiner Rolle als Konsument eine Selbstverpflichtung ein.

Für gerechten Handel mit der Dritten Welt eintreten heisst, als Konsument,

- bewusst umweltverträgliche Produkte kleinbäuerlicher und gewerblicher Herkunft zu bevorzugen ;

- Erzeugnisse aus grossbetrieblichem Anbau und Fertigung nur dann zu berücksichtigen, wenn soziale und ökologische Mindeststandards eingehalten werden:

- durch den Einkauf im Alltag gezielt den Handel mit jenen Produkten zu fördern, welche möglichst viele Arbeitsplätze in den Entwicklungsländern erhalten.

Für gerechten Handel mit der Dritten Welt eintreten heisst für die Wirtschaft,

- beim Aufbau von Produktionsstätten in der Dritten Welt und beim Handel mit den Entwicklungsländern den sozialen und ökologischen Fragen ebensoviel Aufmerksamkeit zu schenken wie der Wirtschaftlichkeit. Im 
langfristigen Eigeninteresse notabene, denn die Löhne von heute sind die Märkte von morgen ;

- im Sinne einer Sozialbilanz und Umweltbilanz der Oeffentlichkeit Rechenschaft abzulegen über die Auswirkungen der Unternehmenstätigkeit in den Entwicklungsländern :

- die Konsumenten ausreichend über das Herkunftsland sowie die Herstellbedingungen der eingeführten Erzeugnisse in sozialer und ökologischer Hinsicht zu informieren.

Für gerechten Handel mit der Dritten Welt eintreten heisst für Bundesrat und Verwaltung,

- sich im Rahmen von internationalen Rohstoffabkommen und anderen Verträgen für existenzsichernde Preise an die Bauern einzusetzen. Es heisst, auf Rohstoffabkommen mit Umwelt- und Sozialklauseln hinzuwirken. Ebenso ist eine vielfältige Wirtschaft in den Entwicklungsländern zu fördern :

- gezielte Handelsabkommen vor allem mit Schwerpunktländern der schweizerischen Entwicklungshilfe abzuschliessen. Gerechte Handelsbeziehungen sind in der Form von Starthilfen vermehrt durch die Entwicklungszusammenarbeit zu fördern :

- den enormen Schwankungen der Rohstoffpreise und Exporterlöse vieler Entwicklungsländer mit allen Mitteln entgegenzuwirken. Schweizerische Initiativen zur Kompensation unverschuldeter Ausfälle der Exporterlöse sind zu entwickeln ;

- auf jegliche Handelsbarrieren, welche die Einfuhr sozial gerechter und umweltverträglicher Produkte behindern, zu verzichten.

Für gerechten Handel mit der Dritten Welt eintreten heisst für unsere eidgenössischen Parlamentarier beispielsweise, im Rahmen des neuen Konsumenteninformationsgesetzes klare rechtliche Grundlagen zu schaffen, um die Konsumenteninformation über Herstellbedingungen und Ursprungsland der eingeführten Produkte wirksam voranzutreiben.

\section{BREITES EINVERSTAENDNIS MIT DER GRUNDIDEE}

Soziale Rücksichtnahme auf Produzenten sowie Information der Konsumenten entsprechen einer guten schweizerischen Tradition:

- 1877 war die Schweiz sehr fortschrittlich, als sie im Fabrikgesetz Normen zum Schutze des Schwächeren, wie das Verbot der Kinderarbeit errichtete.

- Unsere Landwirtschaftspolitik anerkennt seit langem den Anspruch der Schweizer Bauern auf faire Entlöhnung.

- Von 1942 bis 1968 bestand ein Label, d.h. ein Gütezeichen, das Produkte in der Schweiz auszeichnete, die unter gerechten, d.h. sozial fortschrittlichen Bedingungen hergestellt wurden.

- Ein Schweizer Grossverteiler nennt in seiner Sozialbilanz als Ziel seiner 
Einkaufspolitik: "Die Lieferanten sind zur Gewährung vorbildlicher Löhne und Sozialleistungen anzuhalten.»

- Die Schweiz hat mit vielen Ländern bereits Abkommen über den gegenseitigen Schutz von Herkunftsangaben abgeschlossen und ist als Mitglied der Internationalen Arbeitsorganisation an Uebereinkommen über Gewerkschaftsfreiheit und gerechte Entlöhnung beteiligt.

Wohl im Gefolge dieser gut schweizerischen Tradition hat sich Bundespräsident Dr. Alphons Egli in einer Rede am 15. Februar 1986 ganz im Sinne der Aktion «Für gerechten Handel mit der Dritten Welt» geäussert: "Damit die Entwicklungsländer sich selber helfen können, müssen auf den internationalen Güter- und Kapitalmärkten günstige Voraussetzungen geschaffen werden. Dies beinhaltet einmal, dass die Dirttweltländer für ihre Exportprodukte faire Preise erhalten. Preise, die nicht allzu grossen Schwankungen unterliegen, damit eine planmässige und längerfristige Entwicklungspolitik möglich wird. Die Konsumenten in den Industrieländern müssen aber auch bereit sein, diese fairen Preise zu bezahlen.»

Bis zum Welternährungstag am 16. Oktober 1986 haben über 100.000 Menschen den Appell unterschrieben. Die hohe Unterschriftenzahl ist ein Nachweis dafür, dass eine bedeutende Schicht von Konsumenten willens ist, nach sozialen und ökologischen Kriterien einzukaufen. Dieses Ergebnis verdient Beachtung sowohl bei den Konsumentenorganisationen, in der Wirtschaft, bei den Behörden und Politikern.

Dieses Ergebnis soll zu einem Ausgangspunkt für eine neue, auf die Förderung "entwicklungskonformer» Produkte ausgerichtete Strategie werden. Es geht nicht um mehr Handel um jeden Preis, sondern um die gezielte Förderung gerechten Handels. Damit wir nicht unwillentlich zu Profiteuren von Hungerlöhnen oder unwissentlich zu Komplizen der Umweltzerstörung in den Entwicklungsländern werden. Damit sozialgerechte und umweltgerechte Produkte marktgerecht werden. 